The abmitted matuscript has been authored by a contractor of the U.S. Gowernmart. under contrict No, W-3t-109ENG-38. Accordingly, the U. 5 Government retains a nonezelusive, royalty-fres liemm to publish or mproduce the bublithed form of this contribution, or sllaw others to do 10 , for U.S. Govemment purposet.
ANL-HEP-TR- $-90-77$

Refin: DE91 017972

SEP 0619.

SDC-90-00073

24 August 1990

\title{
A First Simulation Study \\ of the Barrel-Endcap Transition Region \\ in a Calorimeter of the Scintillator Tile Design *
}

JAMES Proudfoot and HaNs-Jochen Trost

High Energy Physics Division - 962 -

Argonne National Laboratory

9700 S. Cass Ave., Argonne, IL 60439-4815, USA

\section{ABSTRACT}

We have made a first study of the calorimetric response to $10 \mathrm{GeV} / \mathrm{c}$ charged pions in the transition region between barrel and endcap for the scintillator-tile design pursued at Argonne National Laboratory using the simulation program ANLSIM. For (very nearly) projective tower orientations in the barrel, the crack appears deep within a narrow angular range, causing a loss of the response in that region up to $40 \%$. Pointing the towers onto the beam axis $35 \mathrm{~cm}$ or more away from the nominal.interaction point leads to a shortened depth of the barrelendcap crack as seen by particles incident from the interaction region, cutting the maximum loss down by almost one half. The worsening of the resolution follows the same trend. Introduction of a solenoidal coil in front of the calorimeter causes an overall degradation of the response by an amount nearly comparable to the effect of the crack. Electrons of the same incident momentum are more strongly affected by the coil than pions but see only a much narrower region of degradation by the crack.

* Work supported by the U.S. Department of Energy, Division of High Energy Physics, Contract W-31-109-ENG-38 


\section{Introduction}

Calorimeter designs for SSC detectors are often subject to a variety of mutually contradictory semi-hard requirements and boundary conditions. The underlying demands arise from the physics issues expected to be addressable by SSC experiments. These include hermeticity and homogeneity of the response over most of the solid angle and requirements on position and energy resolutions for electromagnetic and hadronic showers. In particular at the size scale to be considered at the SSC, the technical feasibility tends to create contradictions between those requirements. For instance, good spatial resolution is best achieved with a projective-tower readout geometry. This leads either to projective gaps ruining the hermeticity and local homogeneity of the response or to large cracks between large modules or to large amounts of nonsensitive materials in the region of the shower development.

In this note we present a simulation study of the barrel-endcap transition region for a calorimeter design as pursued at Argonne National Laboratory for a scintillator-tile sampling calorimeter. ${ }^{[3]}$ In the following, we describe the tools and procedures used (section 2) and the results of the simulation runs and conclusions (section 3).

\section{Tools and Procedures}

The starting point for the present study is a design by N.Hill ${ }^{[1]}$ shown in Fig.1. We have coded this geometry as shown in Fig.2 for use in the simulation program ANLSIM, ${ }^{[2]}$ which is based on GEANT. ${ }^{[3]}$ In the azimuthal direction, the setup is simplified for the simulation as being cylindrical everywhere. The tower geometry is coded and handled independently of the GEANT data structures. The simulation program is kept flexible with respect to the design by basing it on a number of parameters measuring various dimensions that can be set at runtime. Thus a design change can be accommodated without additional coding 
and recompilation, within some limits. The relevant parameters are listed in Table 1. In this setup, the coil produces a homogeneous axial magnetic field of $2 T$ strength (dialed by input at run time) in the volume it encloses. There is no flux return modeled, so the calorimeter resides completely in a field free region. The essential features of this design for the present study are detailed in Appendix 1 which may serve as a guide to set the input variables for the program as well.

A set of options are investigated below making use of the selections offered in the program setup:

1. the standard design as given by N.Hill, ${ }^{1 !}$ with 7 towers at the center oriented straight at a right angle to the beam axis, i.e. 3.5 on each side of $z=$ $0 \mathrm{~m}$, four more towers maintaining the radial thickness of the barrel and pointing, like all further barrel towers, to $z=3.5 *$ towerwidth, and endcaps "fitting" into the conical end faces of the barrel with axialiy oriented towers of constant cross section; this is a nearly projective geometry in the barrel, avoiding the gaps between the barrel and the endcaps to point directly to the interaction point;

2. the strictly projective design, different from the standard simply by having no straight central towers;

3. further nonprojective designs like the standard one with the offset of $\mathbf{3 . 5}$ tower widths replaced by $1.5,2,2.5,3,5.5,7.5$ and 20 tower widths.

The variation the offset for projecting the towers determines the projectivity of the crack between the barrel and the endcap. In all cases, the endcap is moved in to just touch the aluminium support cones ( 1 inch thick) of the barrel. This leads to a minimal air gap. No material inside the volume enclosed by the calorimeter is simulated in the round through varying tower offsets; the magnetic field in that volume is taken present nevertheless. The calorimeter response is calibrated in the simulation for electromagnetic and hadronic energy depositions separately to recombine to an $\mathrm{e} / \pi$ ratio of close to 1.0 and to reproduce the absolute energy 
scale, both to within about three percent.

For each setup, program runs are made sending negatively charged pions of momentum $10 \mathrm{GeV} / \mathrm{c}$ into the calorimeter at 14 values of pseudorapidity, $\eta=1.20,1.30,1.34-1.58$ in steps of 0.04 , at 1.80 and intermediate points at $1.40-1.52$, and randomly chosen azimuth $\phi$. The center of the gap between the barrel and the endcap is located around $\eta=1.44$. The pion induced showers are simulated in ANLSIM using full analog simulation with relatively high cuts on the kinetic energies; they are set to $100 \mathrm{MeV}$ to limit the execution times. This will misestimate the absolute resolution somewhat and also slightly reduce the shower size. It will nevertheless give a reasonable relative comparison of the design variants under consideration.

\section{Results on Calorimeter Response}

The total deposited energy before any clustering is performed is taken as the response of the calorimeter for the present purposes. The distributions of this energy normally contain about one thousand entries for each preset value of the pseudorapidity $\eta$. The results for three of the offsets investigated are collected in Table 2.

The calorimeter shows a flat response (Fig.3) of about $10 \mathrm{GeV}$ with a deep dip of observable width in the gap between the barrel and the endcap developing as the tower orientation offset goes towards zero. (The error bars for the means in this figure show the r.m.s. value of the distribution and not the error of the mean, for the r.m.s. values, the error bars have no physical meaning at all.) At the "standard" design offset of $35 \mathrm{~cm}$, the dip is a little more than $20 \%$ for $10 \mathrm{GeV} / \mathrm{c} \pi^{-}$incident, up from $40 \%$ for the projective design, and has begun to level off in dependence on that offset (Fig.4). Adding more material in the gap will widen and deepen the dip in the response curve particularly for (nearly) projective designs. Choosing a point away from the deep-dip region as given by 
the present stucly will then proride some safety nuargin against getting into the large uncertainties connected wiil the strong response variations.

We then inclucle a simple model of the solenoiclal coil in front of the calorimerer as shown in Fig.? using the standard $35 \mathrm{~cm}$ tower projectios offset and study the resulting response to pions (Fig.5). We find an orerall clegradation of more than $10 \%$ within the solid angle corerage of the coil with only half of that effect. in the crack-related dip. Clearly, the coil as modeled provides a scattering center in front of the crack that spreacls the energy cleposition beyond the coil into the sensitive regions of the calorimeter. The range in pseudorapidity affected by the coil end is about $\eta=1.42-1.52$ and the resolution is degracled by less than $30 \%$. This is certainly a somewhat too optimistic picture of the reality since there may be more material in the coil end itself (we use a massive aluminium ring of thickness $15 \mathrm{~cm}$ along the beam axis and $30 \mathrm{~cm}$ radially) and will be more material for support of tracking systems and supplies for everything insicle the rolume enclosed by the calorimeter. For comparison, the simulation efforts for the SDC: Expression of Interest ${ }^{[4]}$ assume the range of clegradation to be $\eta=1.25-1.5$ and the increase of the resolution up to $70 \%$.

An additional check on the performance for the stanclard geometrical choice is to look at the response and acceptance for electrons. We performed rums for the stanclard clesign without and with coil in place for electrons of $10 \mathrm{Ger} / \mathrm{c}$ momentum. For the set and clensity of points in pseudorapiclity used, the crack procluces a rery cleep but also narrow $(\Delta \eta \approx 0.01$ wicle) clip in the response. The coil takes much more energy away from the electrons than from pions as has to be expected (Fig. 0 ) and also worsens the response in the dip significantly: This means the clesign uncler consicleration esscntially will have a narrow ring almost. blind to electrons of moderate energies. This is probably acceptable unless the gap lias to be widened for hardware support installations a lot.

To conclucle, we have obtained support from full simulations of pion showers for a preference of using a quite moderately nonprojective tower geometry in the 
absorber-scintillator tile calorimeter as considered for SDC. The tower orientation determines the orientation of the gaps in the calorimeter coverage, and it is these gaps that have to be well covered by active detectors in a view from the interaction point. The impact of having a solenoidal coil in front of the barrel calorimeter the ends of which reach into the barrel-endcap region is in size comparable to the effect of the crack itself. In the final detector, the crack will contain more material than only the support cones for the barrel calorimeter. For that total amount of passive material, the 1 inch thickness of the support cones is a very crude and possibly thin approximation. In continuing this study, we will look at the impact on particle and jet reconstruction in some detail, and we will aiso attempt to improve the geometry to reflect more realistically the dead material needed by the detectors and the simulation technique to study higher particle energies. 


\section{APPENDIX 1}

The following is a detailed description of the structure of the geometry setup used in ANLSIM:

1. The central towers in the barrel are oriented strictly at a right angle with respect to the beam axis, thus they are not projective in the $(r, z)$ plane (where $r$ denotes the radius in the plane transverse to the beam axis). The number of these towers, counted from $z=0$ to one side only, is given in the variable BARTOF. In the range of these towers the total transverse thickness of the barrel calorimeter is set by the variable BARDRC. The side length of the central towers at their front face is taken to be BARTOW.

2. At the front faces, a conical shell of aluminium of thickness BARCON is attached to support the barrel.

3. By projecting the outside boundary of the central towers onto the beam axis, a secondary origin is defined. From this origin outward, i.e. away from the main coordinate origin, a pseudorapidity variable is used to define the polar boundaries of the remaining barrel towers. A constant stepping in pseudorapidity produces a constant projected size of the towers at the inner surface of the barrel (see appendix 2). The same side length BARTOW is imposed here for the projected size that is used for the central towers also. Thus, a strictly projective tower geometry in the barrel can be produced by having zero central towers as defined above; this is in fact allowed by the simulation program. In turn, an extremely nonprojective geometry can be defined by making the central towers extend over most of the barrel range. This range or half length at the inside surface is defined as the half length of the coil COILZB plus an extension BAREXT over this point, truncated to the largest number of complete towers that can be contained in this bound.

4. Across the first BARTOH towers in the - relative to the secondary origin - projective region, the central transverse thickness of the calorimeter is 
maintained. Beyond those, the outer transverse radius decreases linearily with the axial coordinate $z$ to produce an outer side length BARDRE of the "last" ring of barrel towers. (That length is measured on a ray from the secondary origin along the side face of the towers.)

5. The endcaps are constructed as thick cylinders (length/depth ENDDZC, radial extent ENDDRT starting at an inner radius ENDRIN), with a few (ENDDRI) rings within the radial extent reduced in depth on the inside and a larger number (ENDNST) of rings added on the outside to form an approximation to a conical shape. The inside rings follow a cone with its apex at the primary origin. The outer rings are aligned to have their edges at a constant distance from the outermost ring of barrel towers, i.e. they follow a cone parallel to the cone with an apex slightly further out than the secondary origin and an angle given by the end face of the barrel. The placement of the whole endcaps aling the beam axis is governed by the half length COILZH of the coil and the gap width EINDGAP between the coil and the front face of the endcap. The towers are oriented axially, i.e. not projective; this feature is fixed.

6. The outer rix:- of the endcaps are divided into a short and a long remnant piece each such that the short rings just overlap by a given amount ENDRGO. The idca is to allow to form pseudotowers on the endcap side of the gap between the barrel and the endcap that approximately continue the tower structure of the barrel by one more layer. This could be useful for pattern recognition across that gap. In the present study, this feature has not been used and the short pieces are effectively joint with the longer remnants into one piece.

7. The azimuthal subdivision troughout all of the calorimeter parts is chosen to give a width at the inner face of the calorimeter as close to the desired tower side length (BARTOW and ENDTOW resp.) as possible under the restriction of having an integral number of towers around the azimuthal 
circumference. (This will give an inaccurate model of the performance of pattern recognition in the endcap region; for the current purpose, this does not matter.)

8. The whole calorimeter is subdivided into one longitudinal electromagnetic section of transverse/axial thickness BARDEM and ENDDEM resp., and one hadronic section.

9. Each tower (electromagnetic and hadronic separately) is assigned artificially a coordinate in the pseudorapidity-azimuth plane $(\eta, \phi)$ as follows:

(a) The $\phi$ coordinate is taken at the azimuthal center of the tower in all cases.

(b) The $\eta$ coordinate for the electromagnetic towers is taken at the halfway point into the tower on their center lines.

(c) The $\eta$ coordinate for the hadronic towers in the barrel and in the main body of the endcap is taken 1.5 times the depth of the electromagnetic section (not the length of the tower in front), i.e. BARDEM and ENDDEM resp., into the hadronic section at right angles, not measured along the center lines of the towers.

(d) In the rings of the endcaps, the assigned eta coordinates are picked on cones connecting to the plane used in the main body, with a cone angle taken wide enough to conserve the spatial radial order of rings of towers in the pseudorapidity order.

10. The detector geometry can be extended (via input flags at run time) to include a beam pipe, a coil, a model of an all-scintillating-fiber tracker, and a muon system. The coil parameters are used in the setup of the calorimeter as is evident above; they are available and modifiable even if no actual placement of the coil into the geometry setup to be known by ANLSIM/GEANT is requested. 
The routines needed to define and use the setup are kept in a separate file, replacing the default ones of ANLSIM at linking time.

\section{APPENDIX 2}

The equivalence of constant tower size in the nseudorapidity coordinate $\eta$ and in projected size (side length $2 \cdot \Delta w$ ) at the inside of a cylinder of inner radius $R_{0}$ can be seen as follows:

Let the ray from the interaction point into the center of a tower have the polar angle $\Theta$ with respect to the beam axis. Then the half width $\Delta w$ of the tower is related to a deviation of the polar angle

$$
\Delta w=\frac{R_{0}}{\sin (\Theta)} \tan (\Delta \Theta) .
$$

In order to study the angular dependence of the "cumulated half widths" function $w$, let us use in the limit of small angular deviations the approximation of the tangent function by its argument,

$$
\tan (\Delta \Theta) \rightarrow \Delta \Theta
$$

This substitution leaves the limit $\Delta \Theta \rightarrow 0$ of $\Delta w$ invariant, being

$$
\frac{d w}{d \Theta}=\frac{R_{0}}{\sin (\Theta)}
$$

as the difference $\tan (\Delta \Theta)-\Delta \Theta$ is continuously differentiable in a whole neighborhood of zero and it and its first derivative vanish at zero (the former quadratically as its Taylor series exists and converges in that region). By integration from $\Theta=\pi / 2$ to any polar angle we find ${ }^{[3]}$ for the function $w$ the representation

$$
w(\Theta)=R_{0} \ln \left(\tan \left(\frac{\Theta}{2}\right)\right)=-R_{0} \eta .
$$

Thus, the request arising from the physics of jets to define towers with constant width on the (pseudo-) rapidity scale and that one arising from pattern recognition to define towers of constant width across the lateral extension of showers 
actually coincide in a cylindrical calorimeter aligned on the beam axis. This coincidence is lost in the endcaps where $\Delta w$ has a different dependence on $\Theta$.

\section{DISCLAIMER}

This report was prepared as an account of work sponsored by an agency of the United States Government. Neither the United States Government nor any agency thereof, nor any of their employees, makes any warranty, cxpress or implied, or assumes any legal liability or responsibility for the accuracy, completeness, or usefulness of any information, apparatus, product, or process disclosed, or represents that its use would not infringe privately owned rights. Reference herein to any specific commercial product, process, or service by trade name, trademark, manufacturer, or otherwise does not necessarily constitute or imply its endorsement, recommendation, or favoring by the United States Government or any agency thereof. The views and opinions of authors expressed herein do not necessarily state or reflect those of the United States Government or any agency thereof. 


\section{REFERENCES}

1) N.Hill, Observations and conclusions drawn from conceptual design exercises on barrel calorimeter for SDC, internal note, Argonne National Laboratory, May 30, 1990 (unpublished)

2) R.E.Blair, L.E.Price and H.-J.Trost, program ANLSIM, Argonne National Laboratory, Argonne, Illinois, USA 1989, version 1.04, 1990, unpublished;

H.-J.Trost, talk given at: Workshop on the Future Development of GEANT, SSC Laboratory, Dallas, Texas, Jan.10-13, 1990, unpublished; R.E.Blair, in: Workshop on Physics and Detector Simulation for SSC Experiments, SSC Laboratory, Dallas, Texas, Jan.9-19, 1990, vol.1, p.69; H.-J.Trost, ibid., vol.2, p.265

3) R.Brun et al., GEANT 3, CERN DD/EE/84-1, version 3.13, CERN, Geneva, Switzerland 1990

4) Solenoid Detector Collaboration, Expression of Interest to Construct and Operate a Detector at the SSC, May 24, 1990

5) I.N.Bronshtein and K.A.Semendyayev, Ilandbook of Mathematics, 3rd English edition, Van Nostrand Reinhold Company, New York, New York, USA 1985, section 1.1.3 


\section{TABLE CAPTIONS}

1. Design parameters used in the geometryusetup for ANLSIM (lengths in cm; defaults in parentheses)

2. Pseudorapidity, polar angle, momentum components, mean response and r.m.s. of the response for a) the standard design, b) the projective design, c) the highly nonprojective design with $2 m$ offset

\section{FIGURE CAPTIONS}

1. Side view of the calorimeter design addressed in this study

2. Side view of the mechanical calorimeter geometry as seen by the ANLSIM program - standard design with optional coil

3. Mean response and resolution for $10 \mathrm{GeV} / \mathrm{c} \pi^{-}$of the calorimeter of ref. 1 for different offsets of the tower orientation; the errors on the mean shown are the resolutions, and the error bars shown for the resolution have no physical meaning

4. Minimum response to $10 \mathrm{GeV} / \mathrm{c} \pi^{-}$in dependence on the tower projection offset; the additional star for $35 \mathrm{~cm}$ offset shows the effect of the solenoidal coil.

5. Mean response and resolution for $10 \mathrm{GeV} / \mathrm{c} \pi^{-}$of the calorimeter of ref.1 without and with coil in place

6. Mean response and resolution for $10 \mathrm{GeV} / \mathrm{ce} e^{-}$of the calorimeter of ref.1 without and with coil in place 


\section{Table 1}

PIPEIN

PIPEDR

COILIN

COILMI

COILUT

COILZH

COILEN

COILD1

COILD2

COILD3

BAREXT

BARGAP

BARDRC

BARTOF

BARTOH

BARDRE

BARDEM

BARTOW

BARCON

ENDGAP

ENDRIN

ENDDRT

ENDDRI

ENDDZI

EYDDZC

ENDDEM

ENDRGO

ENDTOW

ENDWST

inner radius of Beryllium beam pipe (4.9)

thickness of beryllium beam pipe (0.1)

inner radius of inner coil shell (185.0)

inner radius of central coil shell (198.5)

outer (!) radius of outer coil shell (215.0)

axial half length of coil (450.0)

axial length of massive coil end pieces (10.0)

radial thickness of inner coil shell (3.0)

radial thickness of central coil shell (3.0)

radial thickness of outer coil shell (3.0)

axial extension on each side of the front face of

the barrel calorimeter (5.0)

radial space between outside radius of coil and

the barrel calorimeter (10.0)

total radial thickness of barrel at $z=0$ (234.9)

number of towers from center out that have constant width in $z$ (3.5)

number of additional towers beond the constant width ones for which the radial thickness of the barrel remains constant (4.0)

total depth of barrel along side to end cap

(243.8)

thickness of electromagrietic barrel section

$(19.3=7.592 * 2.54)$

projected azimuthal front width of towers (10.0)

thickness of aluminium support cone at end faces (2.5)

space between coil end and front face of end cap $(25.0)$

inner radius at front face of end cap (50.0)

radial thickness of end cap at front face (180.0)

radial number of inner towers shorter than full

axial thickness of end cap (3.0)

length of innermost hadronic endcap towers (12.8)

axial thickness of end cap (278.2 $=375 * 0.292 * 2.54)$

thickness of electromagnetic end cap section

$(19.3=7.592 * 2.54)$

axial overlap of rings $(2.3=3 * 0.292 * 2.54)$

side length of end cap tower (10.0)

number of steps for outside rings of end caps (11) 
Table 2a

\begin{tabular}{lcccccc} 
eta & $\begin{array}{c}\text { theta } \\
\text { [degrees] }\end{array}$ & $\begin{array}{c}\mathrm{pz} \\
\text { [GeV/c] }\end{array}$ & $\begin{array}{c}\mathrm{pT} \\
\text { number } \\
\text { of events }\end{array}$ & $\begin{array}{c}\langle\mathrm{E} \text { (obs.)〉 } \\
\text { [GeV] }\end{array}$ & $\begin{array}{c}\text { r.m.s. } \\
\text { [GeV] }\end{array}$ \\
\hline 0.00 & 90.00 & 0.000 & 10.000 & 1000 & 10.16 & 1.25 \\
1.20 & 33.50 & 8.337 & 5.523 & 1000 & 10.17 & 1.20 \\
1.30 & 30.50 & 8.617 & 5.074 & 1000 & 10.10 & 1.30 \\
1.34 & 29.35 & 8.717 & 4.901 & 1000 & 10.14 & 1.21 \\
1.38 & 28.24 & 8.810 & 4.732 & 1000 & 10.04 & 1.26 \\
1.40 & 27.71 & 8.854 & 4.649 & 1000 & 10.12 & 1.32 \\
1.41 & 27.44 & 8.875 & 4.608 & 1000 & 10.05 & 1.28 \\
1.42 & 27.18 & 8.896 & 4.567 & 1000 & 10.07 & 1.24 \\
1.43 & 26.92 & 8.917 & 4.527 & 1000 & 9.91 & 1.39 \\
1.44 & 26.66 & 8.937 & 4.487 & 1000 & 9.41 & 1.39 \\
1.45 & 26.40 & 8.957 & 4.447 & 1000 & 7.72 & 1.97 \\
1.46 & 26.15 & 8.977 & 4.407 & 1000 & 9.44 & 1.57 \\
1.47 & 25.90 & 8.996 & 4.368 & 1000 & 9.79 & 1.31 \\
1.48 & 25.65 & 9.015 & 4.328 & 1000 & 9.92 & 1.30 \\
1.49 & 25.40 & 9.033 & 4.290 & 1000 & 10.00 & 1.32 \\
1.50 & 25.16 & 9.051 & 4.251 & 1000 & 10.04 & 1.26 \\
1.52 & 24.67 & 9.087 & 4.175 & 1000 & 10.14 & 1.26 \\
1.54 & 24.20 & 9.121 & 4.099 & 1000 & 10.13 & 1.25 \\
1.58 & 23.28 & 9.186 & 3.952 & 1000 & 10.14 & 1.32 \\
1.80 & 18.77 & 9.468 & 3.218 & 1000 & 10.18 & 1.26
\end{tabular}


Table 2b

\begin{tabular}{|c|c|c|c|c|c|c|}
\hline eta. & $\begin{array}{c}\text { theta } \\
\text { [degrees] }\end{array}$ & $\begin{array}{l}\mathrm{pz} \\
{[\mathrm{G}}\end{array}$ & $\begin{array}{l}\mathrm{pT} \\
\mathrm{V} / \mathrm{c}]\end{array}$ & $\begin{array}{l}\text { number } \\
\text { of events }\end{array}$ & $\begin{array}{c}\langle E \text { (obs.) }\rangle \\
{[\mathrm{GeV}]}\end{array}$ & $\begin{array}{l}\text { r.m.s. } \\
\text { [GeV] }\end{array}$ \\
\hline 0.00 & 90.00 & 0.000 & 10.000 & 1000 & 10.16 & 1.16 \\
\hline 1.20 & 33.50 & 8.337 & 5.523 & 1000 & 10.09 & 1.40 \\
\hline 1.30 & 30.50 & 8.617 & 5.074 & 1000 & 10.09 & 1.41 \\
\hline 1.34 & 29.35 & 8.717 & 4.901 & 1000 & 10.13 & 1.35 \\
\hline 1.38 & 28.24 & 8.810 & 4.732 & 1000 & 10.15 & 1.39 \\
\hline 1.40 & 27.71 & 8.854 & 4.649 & 1000 & 9.94 & 1.32 \\
\hline 1.41 & 27.44 & 8.875 & 4.608 & 1000 & 9.85 & 1.37 \\
\hline 1.42 & 27.18 & 8.896 & 4.567 & 1000 & 6.95 & 2.08 \\
\hline 1.43 & 26.92 & 8.917 & 4.527 & 1000 & 6.06 & 3.27 \\
\hline 1.44 & 26.66 & 8.937 & 4.487. & 1000 & 9.26 & 1.60 \\
\hline 1.45 & 26.40 & 8.957 & 4.447 & 1000 & 9.73 & 1.51 \\
\hline 1.46 & 26.15 & 8.977 & 4.407 & 1000 & 10.04 & 1.31 \\
\hline 1.47 & 25.90 & 8.996 & 4.368 & 1000 & 10.02 & 1.29 \\
\hline 1.48 & 25.65 & 9.015 & 4.328 & 1000 & 10.00 & 1.33 \\
\hline 1.49 & 25.40 & 9.033 & 4.290 & 1000 & 10.13 & 1.34 \\
\hline 1.50 & 25.16 & 9.051 & 4.251 & 1000 & 10.14 & 1.39 \\
\hline 1.52 & 24.67 & 9.087 & 4.175 & 1000 & 10.15 & 1.22 \\
\hline 1.54 & 24.20 & 9.121 & 4.099 & 1000 & 10.20 & 1.28 \\
\hline 1.58 & 23.28 & 9.186 & 3.952 & 1000 & 10.11 & 1.29 \\
\hline 1.80 & 18.77 & 9.468 & 3.218 & 1000 & 10.15 & 1.31 \\
\hline
\end{tabular}


Table 2c

eta theta $\mathrm{pz} \quad \mathrm{pT}$ number $\langle\mathrm{E}$ (obs.)〉 I.m.s. [degrees] [GeV/c] of events [GeV] [GeV]

$\begin{array}{llrrrrr}0.00 & 90.00 & 0.000 & 10.000 & 1000 & 10.21 & 1.26 \\ 1.20 & 33.50 & 8.337 & 5.523 & 1000 & 10.15 & 1.31 \\ 1.30 & 30.50 & 8.617 & 5.074 & 1000 & 10.22 & 1.32 \\ 1.34 & 29.35 & 8.717 & 4.901 & 1000 & 10.10 & 1.28 \\ 1.38 & 28.24 & 8.810 & 4.732 & 1000 & 9.91 & 1.37 \\ 1.40 & 27.71 & 8.854 & 4.649 & 1000 & 9.78 & 1.33 \\ 1.41 & 27.44 & 8.875 & 4.608 & 1000 & 9.70 & 1.42 \\ 1.42 & 27.18 & 8.896 & 4.567 & 1000 & 9.55 & 1.57 \\ 1.43 & 26.92 & 8.917 & 4.527 & 1000 & 9.72 & 1.43 \\ 1.44 & 26.66 & 8.937 & 4.487 & 1000 & 9.96 & 1.39 \\ 1.45 & 26.40 & 8.957 & 4.447 & 1000 & 10.06 & 1.29 \\ 1.46 & 26.15 & 8.977 & 4.407 & 1000 & 9.99 & 1.34 \\ 1.47 & 25.90 & 8.996 & 4.368 & 1000 & 10.08 & 1.36 \\ 1.48 & 25.65 & 9.015 & 4.328 & 1000 & 10.12 & 1.21 \\ 1.49 & 25.40 & 9.033 & 4.290 & 1000 & 10.11 & 1.25 \\ 1.50 & 25.16 & 9.051 & 4.251 & 1000 & 10.09 & 1.29 \\ 1.52 & 24.67 & 9.087 & 4.175 & 1000 & 10.18 & 1.26 \\ 1.54 & 24.20 & 9.121 & 4.099 & 1000 & 10.18 & 1.21 \\ 1.58 & 23.28 & 9.186 & 3.952 & 1000 & 10.06 & 1.25 \\ 1.80 & 18.77 & 9.468 & 3.218 & 1000 & 10.20 & 1.22\end{array}$




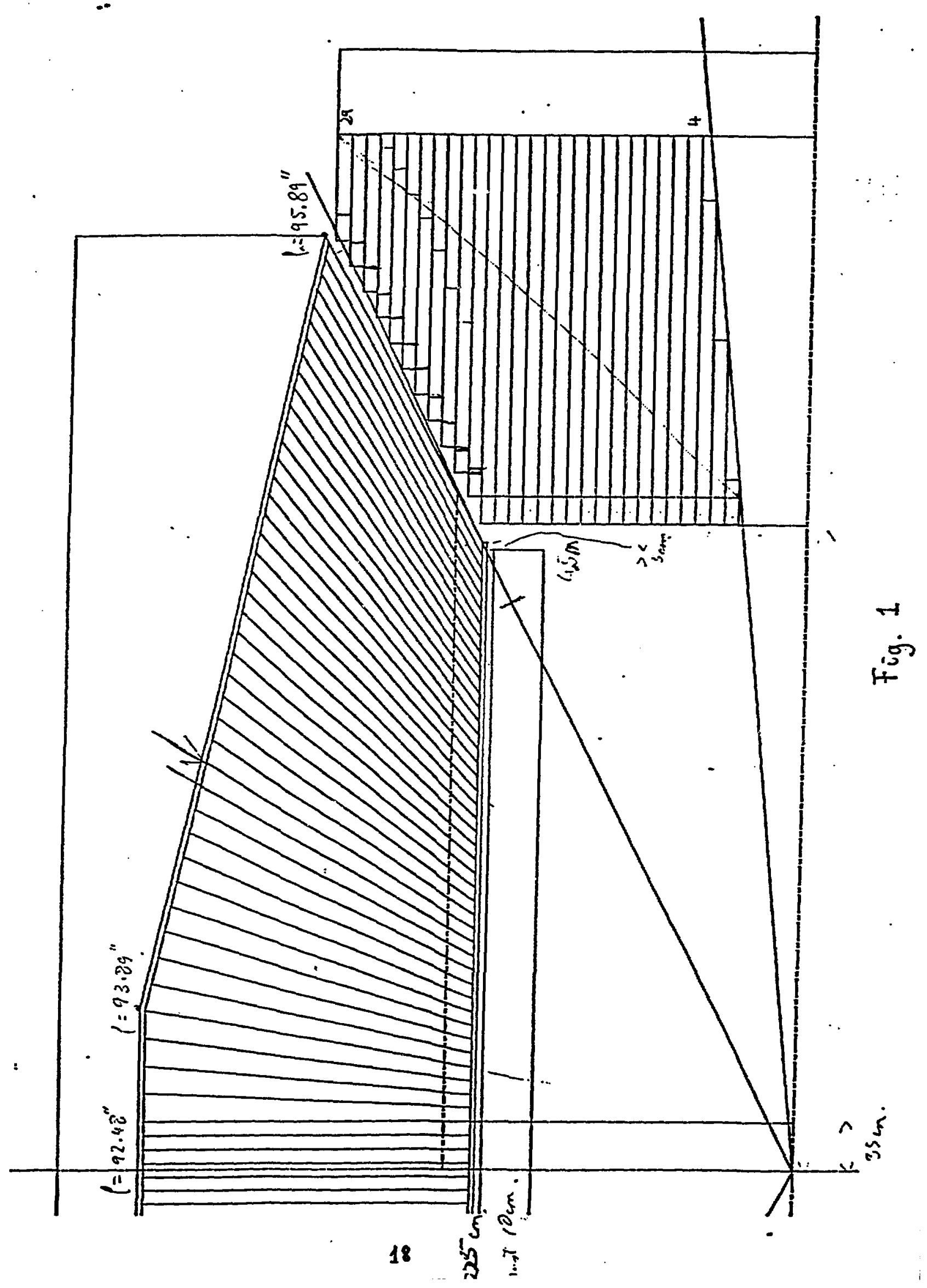




$$
A^{P}
$$


Colorimeter response over $\eta$
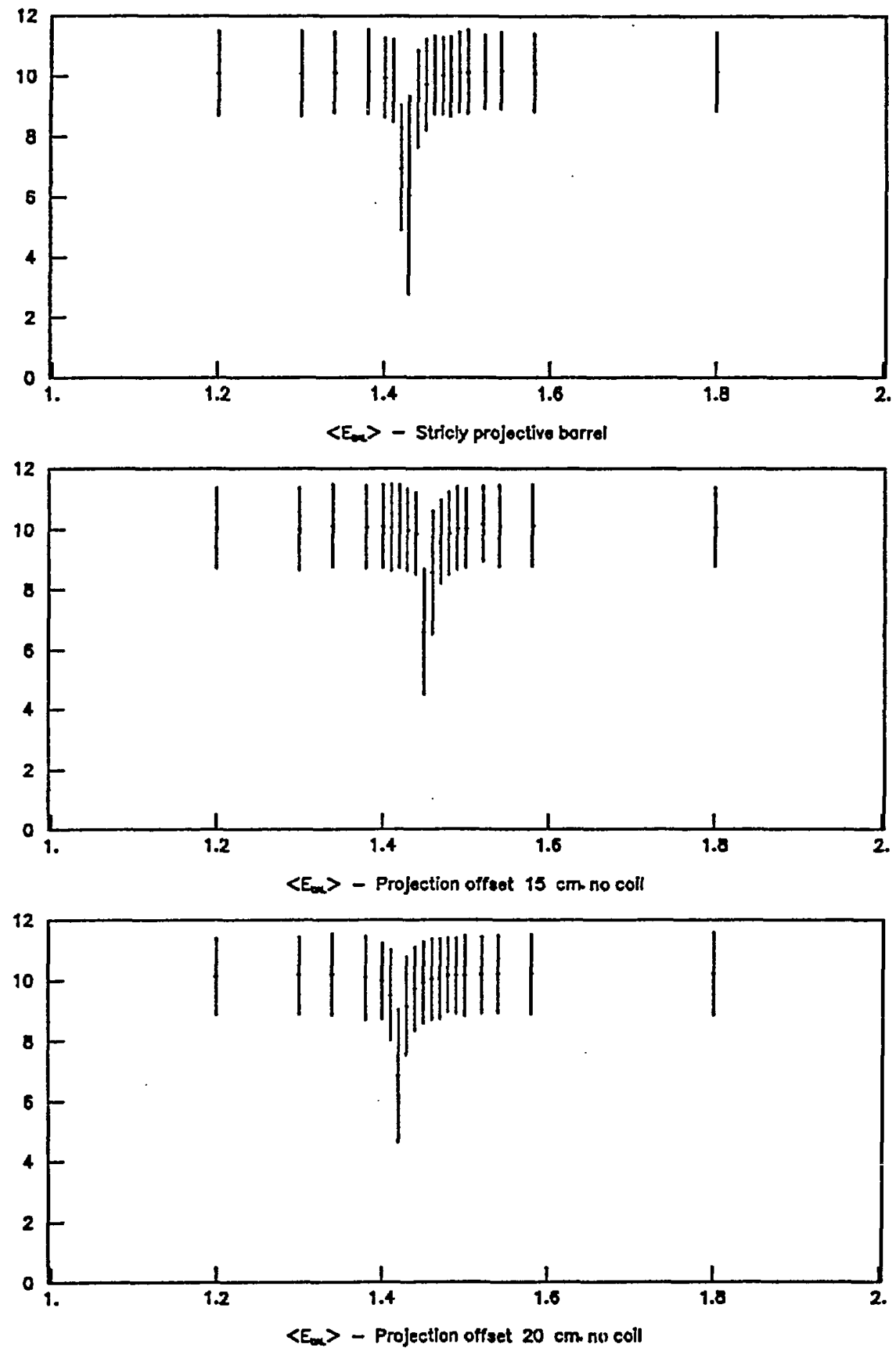

$$
\text { Fig. }_{20}(1 \text { of } 6)
$$


Calorimeter response over $\eta$
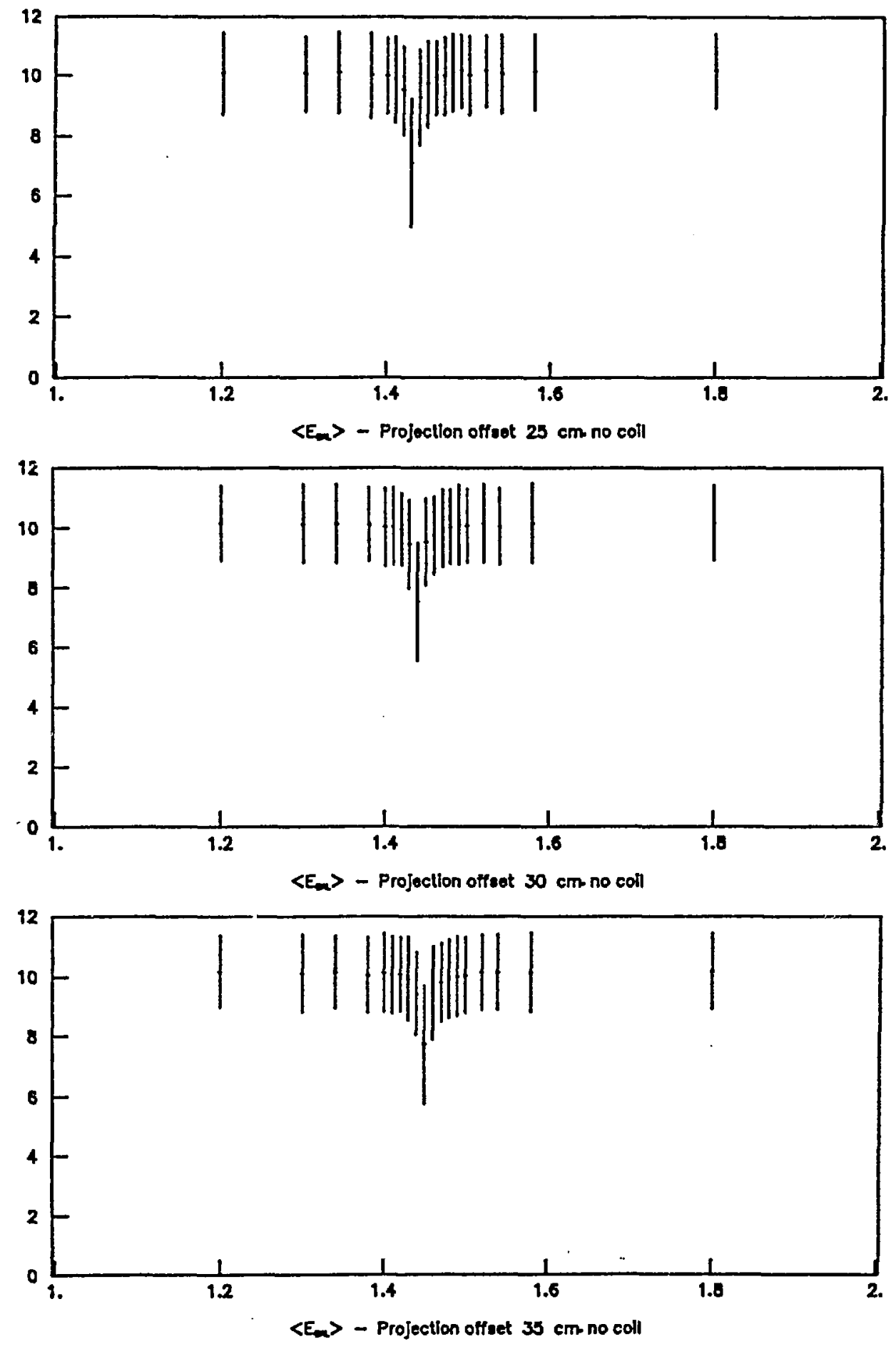

Fig. 3 (2 of 6$)$ 
Calorimeter response over $\eta$
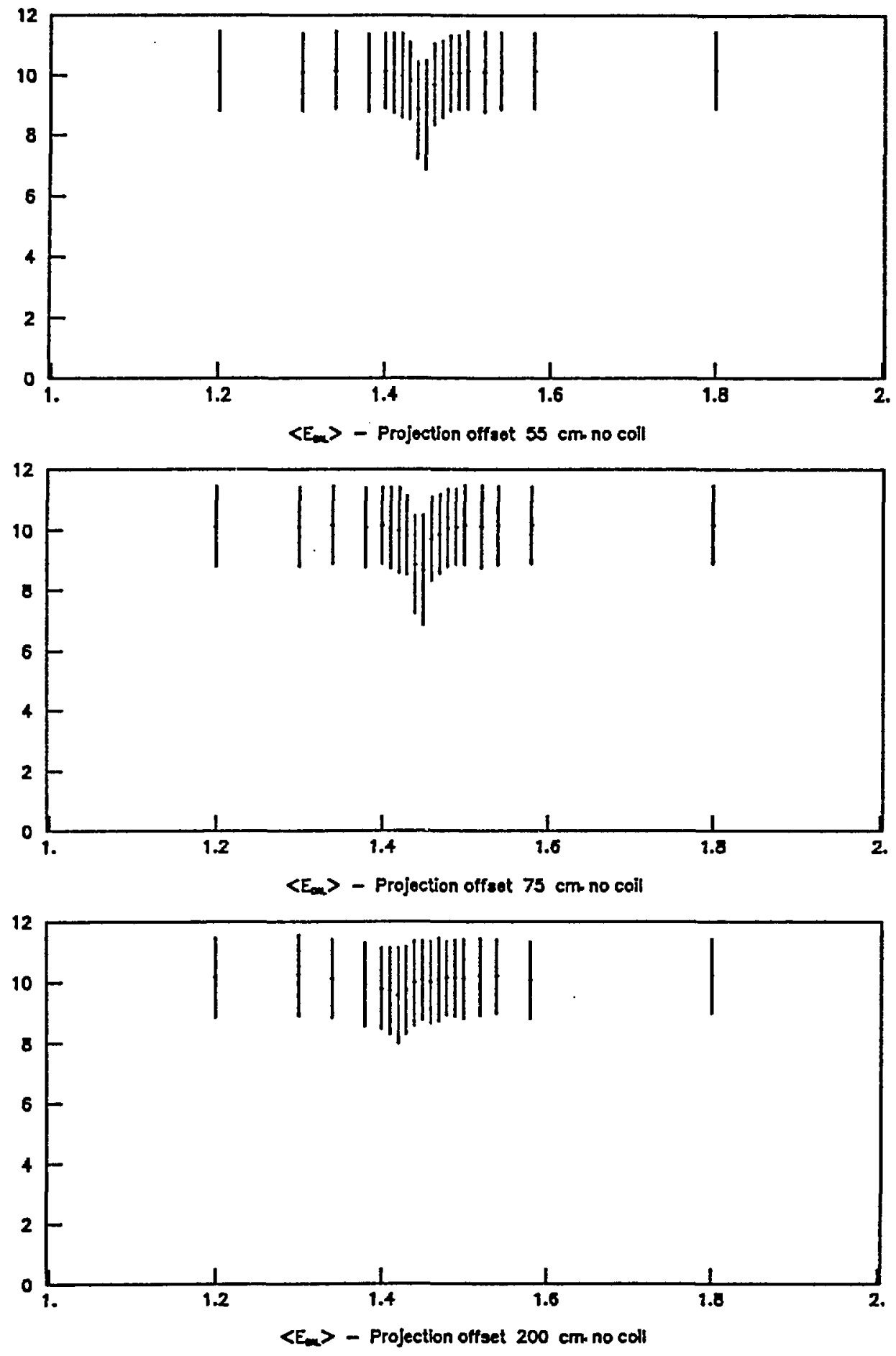

$$
\underset{22}{\text { Fig. } 3(3 \circ f 6)}
$$


Colorimeter response over $\eta$
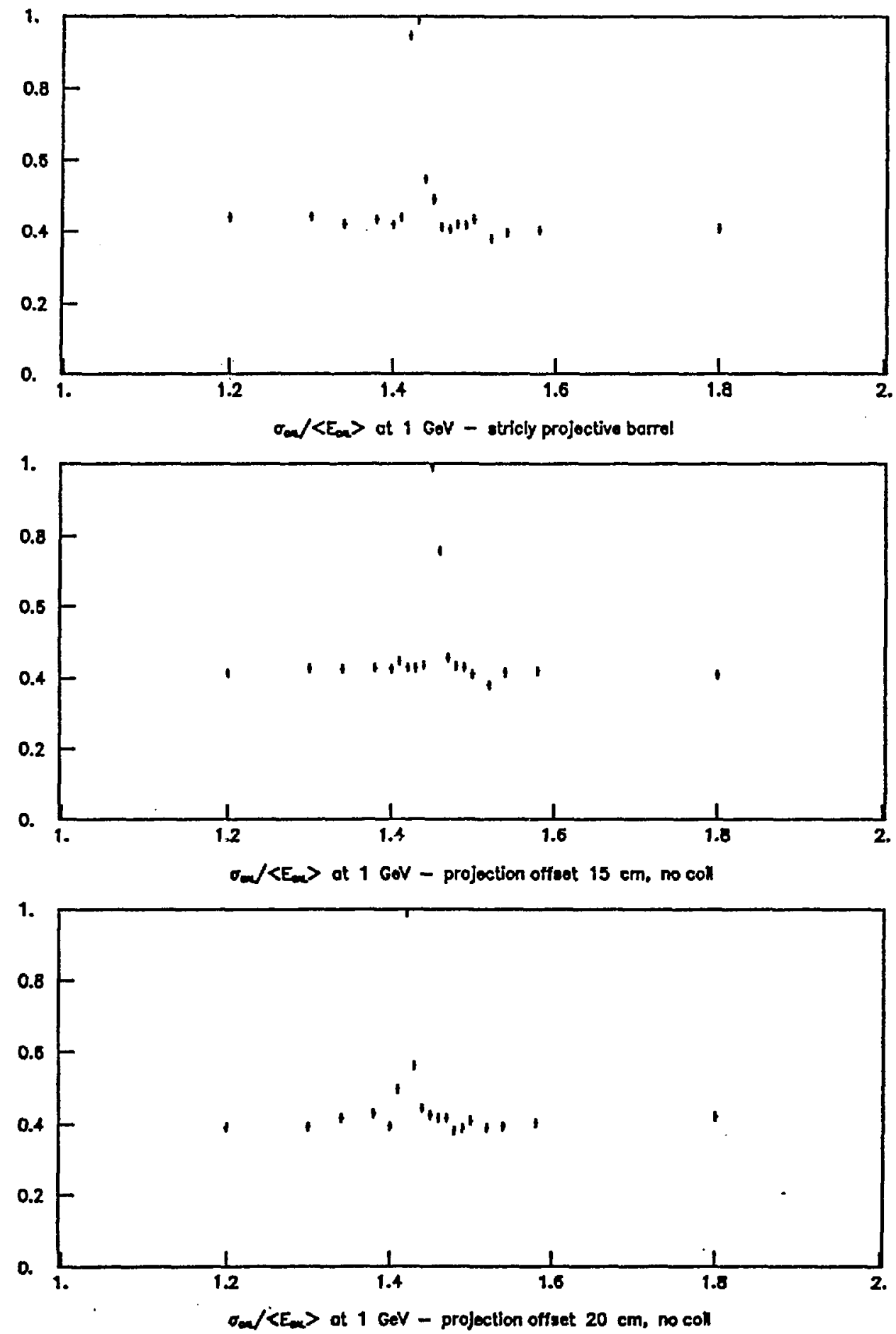

Fig. 3 ( 4 of 6$)$ 
Colorimeter response over $\eta$
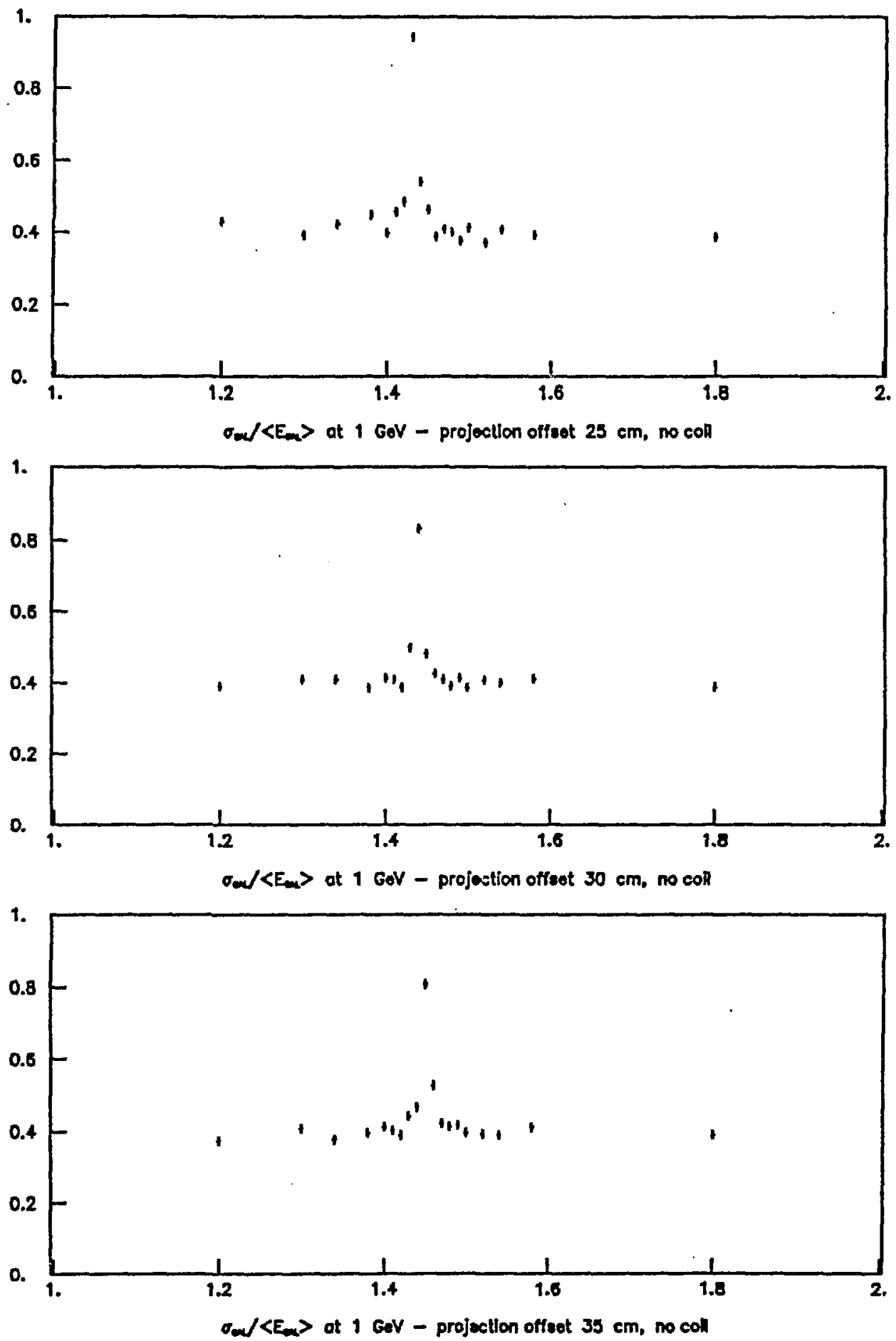

Fig. $3(5 \circ 6)$ 
Calorimeter response over $\eta$
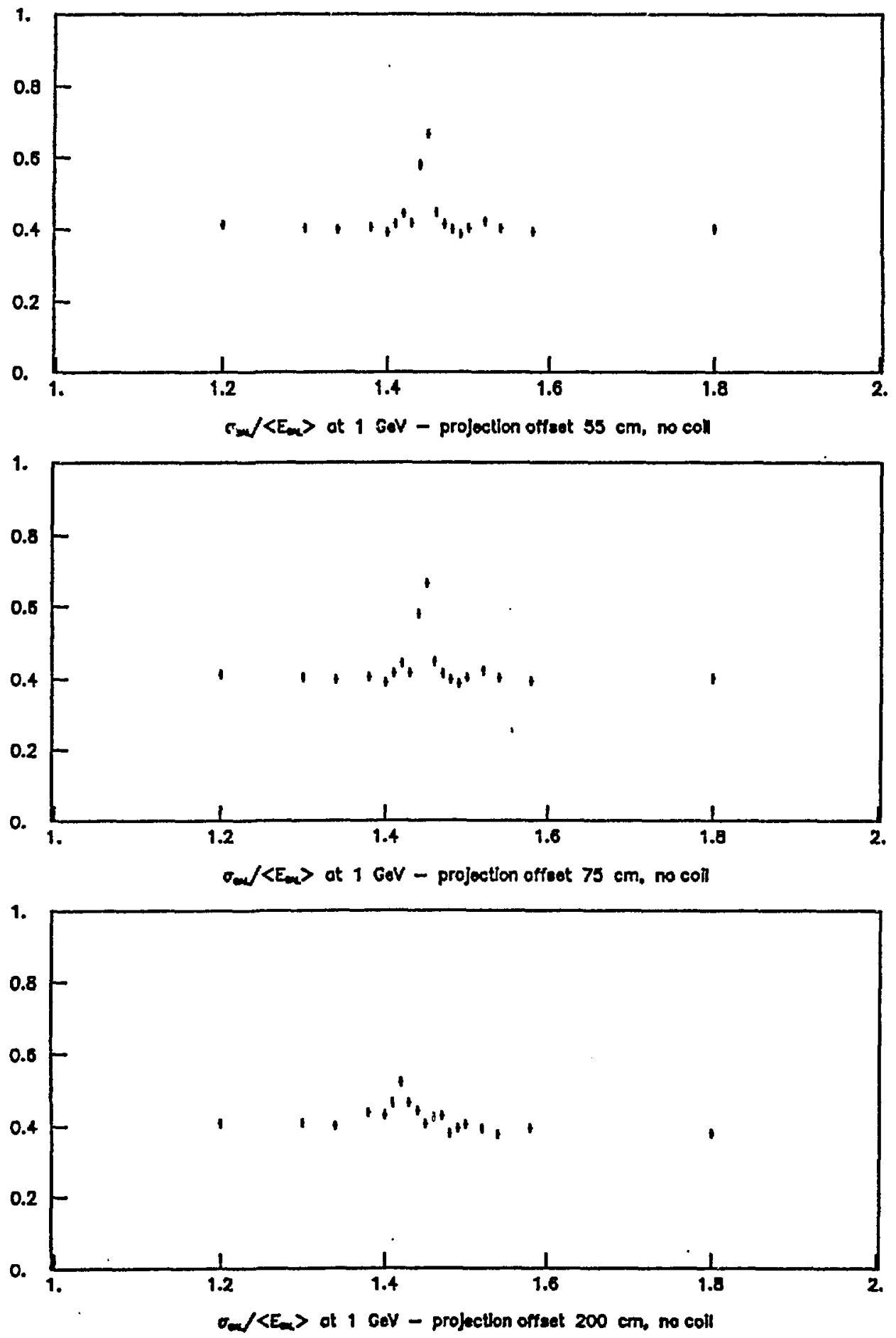

Fig., 3 (6ot6) 
Minimal response in transition region

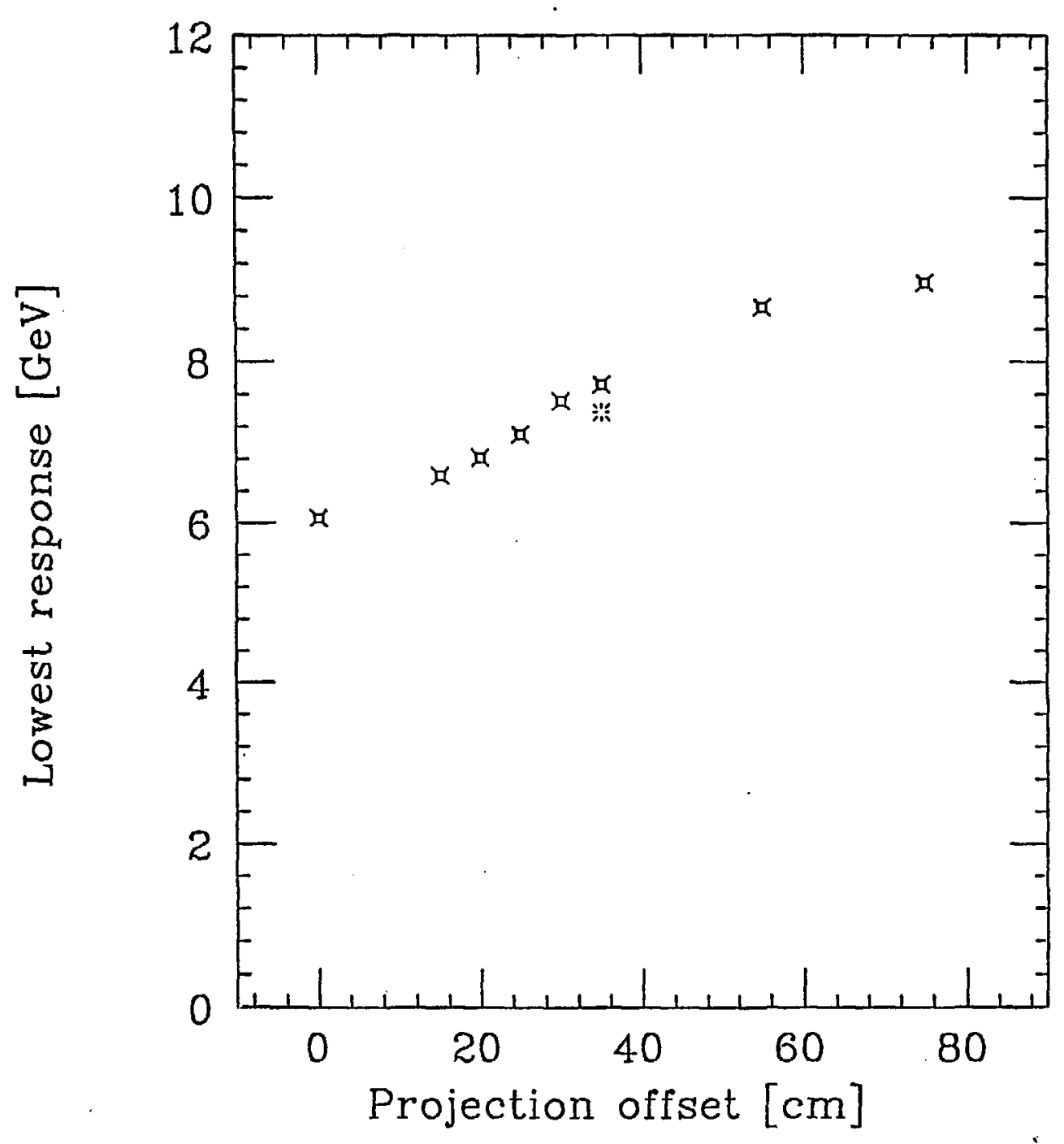

Fig. 4 


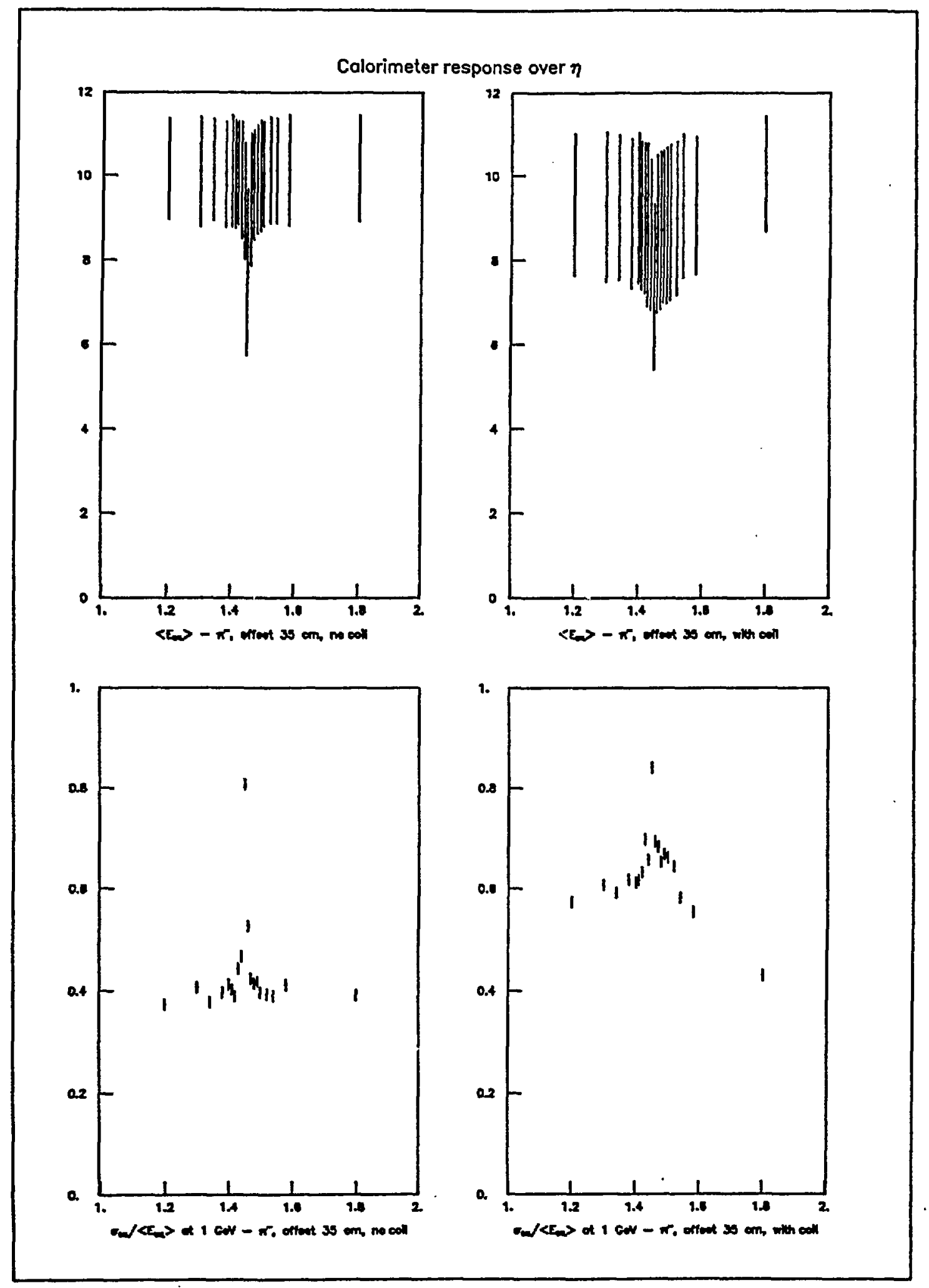

Fig. 5 
Colorimeter response over $\boldsymbol{\eta}$
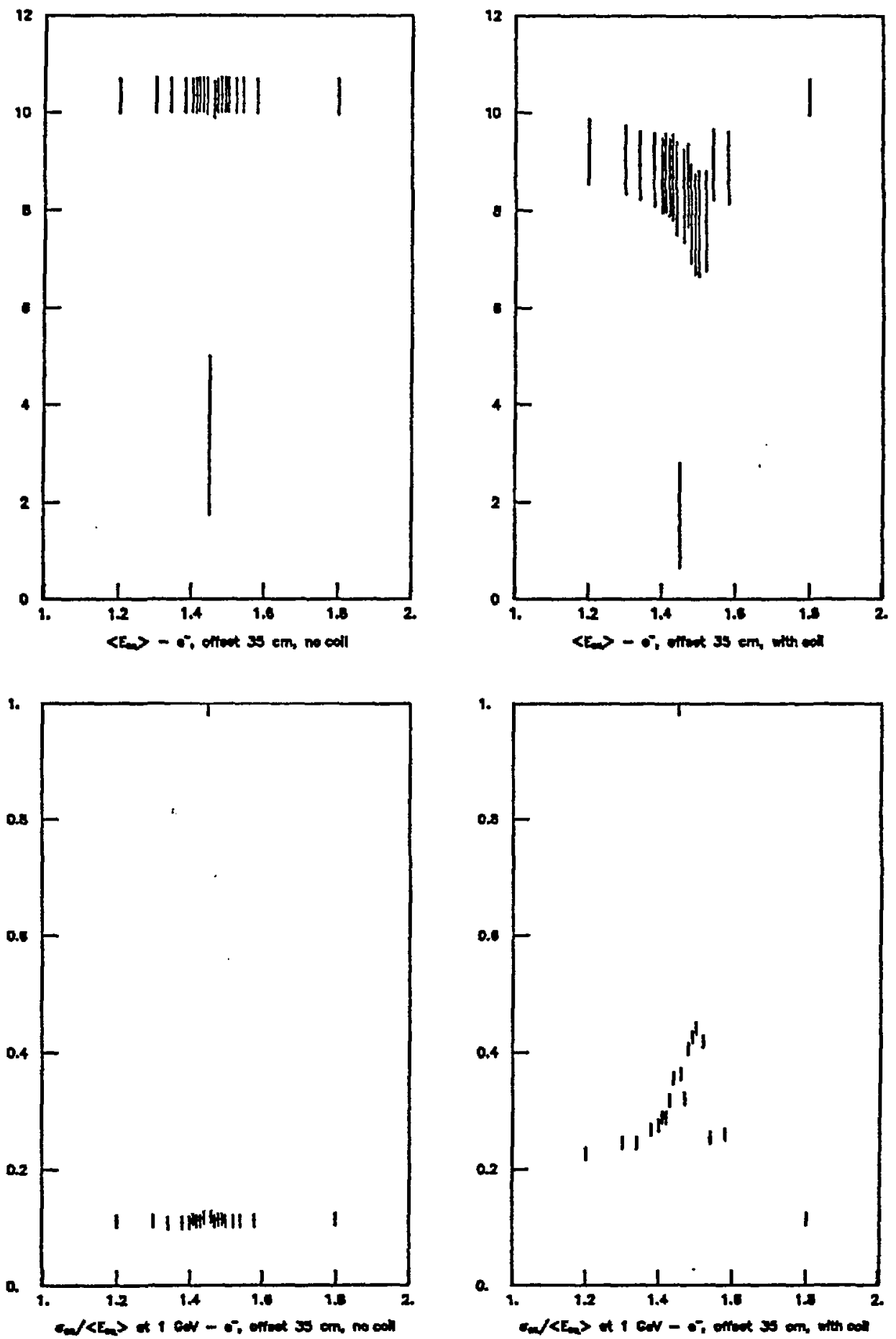

Fig. 6 